\title{
Enunciación
}

http://revistas.udistrital.edu.co/ojs/index.php/enunc

\section{La lectura de los clásicos en la etapa preuniversitaria: una propuesta de intervención}

\author{
The reading of classics in the pre-university stage: a proposal for intervention \\ María José Molina García ${ }^{1}$
}

Para citar este artículo: Molina, M. J. (2015). La lectura de los clásicos en la etapa preuniversitaria: una propuesta de intervención. Enunciación, 20(1), pp. 26-38.

Recibido: 10-mayo-2015 / Aprobado:15-junio-2015

\begin{abstract}
Resumen
En este artículo se plantea el fomento de la lectura de clásicos entre los alumnos preuniversitarios, mediante una propuesta de trabajo basada en la obra dramática de Federico García Lorca, Yerma, por la relevancia y actualidad de los temas que trata.
\end{abstract}

Se presentan los resultados de una investigación cualitativa con la cual se demuestra que a través de la literatura se generan espacios para que los estudiantes imaginen, jueguen con el texto literario, desarrollen una competencia literaria, reflexionen y construyan experiencias con el grupo, y analicen una realidad en donde hay muchas culturas y distintas opiniones. Como novedad, se incluye una perspectiva metodológica bimodal que enfatiza la pertinencia de este texto literario en la sociedad del siglo XXI.

Palabras clave: competencia literaria, competencia intercultural, investigación cualitativa, metodología bimodal, educación preuniversitaria.

\begin{abstract}
This article addresses the promotion of reading classics among pre-university students with the presentation of a work proposal from the play by Federico Garcia Lorca, Yerma, due to the relevance and timeliness of the topics covered.
\end{abstract}

The qualitative research results presented demonstrate that literature creates spaces that allow students to imagine, believe possible worlds, share their reading experiences with the group and play with the literary text. In addition, students develop a literary competence, reflect with the group and discuss a reality where there are many cultures and different opinions. Furthermore, a bimodal methodological perspective that emphasizes the relevance of this literary text in the XXI century is included

Keywords: literary competence, intercultural competence, qualitative research, bimodal methodological, pre-university education.

1 Doctora en Filología y profesora del Departamento de Didáctica de la Lengua y la Literatura de la Universidad de Granada. Correo electrónico: mjose@ugr.es 


\section{INTRODUCCIÓN}

Uno de los problemas más graves con el que nos enfrentamos todos los que trabajamos en el campo de la educación es la falta de interés de nuestro alumnado hacia la lectura. Esto nos lleva a reflexionar y a enumerar las causas de esta deficiencia, muchas de las cuales escapan a nuestras posibilidades como educadores. Lo que sí está a nuestro alcance es tratar de superar, de alguna forma, esta falta de interés. Nuestro objetivo es transmitir que leer merece la pena (Monson, 1989) y tratar de crear un hábito lector que produzca placer y motive a nuestros estudiantes (Gómez-Villalba, 1995, Colomer, 1999; Molina-García, 2002).

El problema se acentúa cuando nuestros alumnos son adolescentes, pues, durante las primeras etapas de la escolaridad, la actividad lectora en general y la expresión dramática en particular, están asociadas al juego y a las técnicas de animación y confieren a la lectura un carácter lúdico muy motivador, el cual va desapareciendo, a medida que avanzan los cursos, dando paso a otra serie de tareas menos estimulantes que desmotivan y desalfabetizan (Jolibert y Gloton, 1985). Lo preocupante es que, según los expertos, un estudiante que termine la etapa de enseñanza reglada sin haber generado una sensibilidad por la lectura, es improbable que fomente este hábito en el futuro.

Pero, ¿hay que obligar a leer? No, la lectura no puede plantearse como obligación porque perderemos la esperanza de que sea una actividad placentera; tampoco hay que acompañarla siempre de tediosas tareas de sistematizar una serie de ejercicios individuales a posteriori que le resten protagonismo y que nos alejen del objetivo fijado: crear el hábito lector. Lo que sí es pertinente es debatir, crear foros literarios en el aula en un clima distendido y reflexionar entre todos sobre lo leído. De esta forma cobra sentido la dimensión social de la lectura y estableceremos espacios para compartirla. Así otorgaremos a la literatura su verdadero papel en el currículum. Si a ello añadimos la aproximación a los nuevos lenguajes digitales, estableceremos los vínculos y puentes necesarios para crear sinergias con el público juvenil. En esta línea, a menudo se sentencia que los jóvenes de hoy en día no leen; pero no es correcto. Lo que ocurre es que, según Cassany (2012), leer se ha convertido en una actividad cada vez más tecnológica. Ahora se leen blogs, revistas, presentaciones, se busca en YouTube, en las páginas de los autores y todo ello se complementa con la lectura del texto impreso, es decir, la lectura ha dejado de ser exclusivamente individual para ser compartida, también, como actividad social. Y de este aspecto podemos hacer eco en las aulas para añadir un valor al hábito lector.

¿Cómo leer? Estamos en la línea de entender la lectura como un acto de comunicación especial (Molina-García, 2002; Cerrillo, 2013) que afecta al conjunto de elementos involucrados en él. Quedan ya lejos, desde un punto de vista ideológico, las décadas del sesenta y setenta en las que se creía en el precepto de que leer era sinónimo de decodificar. Obviamente, para realizar una buena lectura es imprescindible que la decodificación no plantee problemas, pero no es el único aspecto implicado. El profesor que guíe a sus alumnos en el análisis de un texto, debe dirigir su actuación hacia una comprensión lectora. Esta no es un conjunto de habilidades sino un proceso en el que el lector elabora el significado de la información que recibe, apelando a una serie de habilidades o aspectos o claves, y relacionándolas con sus propias experiencias y conocimientos previos (Gómez-ViIlalba, 1995). Se trata de establecer una interacción entre el lector y el libro (Cooper, 1990; Puente, 1991; Solé, 1992; Camps, 1998). Leer es mantener un diálogo interactivo que busque la comprensión y la interpretación (Mendoza, 1998). De ahí que puedan definirse varios tipos de comprensión o varios grados para alcanzar (según la edad de los alumnos): la comprensión literal, la interpretativa, la evaluativa o crítica, y la apreciativa (Vallés, 1991). No vamos a detenernos a explicar cada una de ellas, aunque sí diremos que están implícitas en la propuesta didáctica que exponemos a modo de 
ejemplo, haciendo especial énfasis en la práctica de realizar inferencias, suposiciones, asociaciones, opiniones, identificación de hechos y detalles relevantes, fomento de las normas y características básicas de tipología textual mediante la interpretación e identificación de marcas textuales, así como la utilización de las claves contextuales para la adquisición de vocabulario, etc.; es decir, aquellos aspectos que, a nuestro entender, facilitan la comprensión lectora y que eran obviados en los, ya obsoletos, comentarios de textos. Creemos, además, que con este tipo de práctica docente, se facilita la comunicación oral, esencial en educación.

Por otro lado, entre los instrumentos que la mayoría tenemos siempre a disposición, además de los tradicionales, está el acceso a internet (mediante teléfono móvil, tableta digital, ordenador...) para localizar cualquier tipo de información o como soporte escrito para nuestras producciones. En cuanto a lo primero, sin duda, internet nos libera de la memorización de datos pero, a cambio, exige el desarrollo de unas habilidades y competencias porque, según Marc Prensky (2010), pese a que se diga que las nuevas generaciones, los nativos digitales, son muy hábiles para utilizar las tecnologías de la información y las comunicaciones (TIC), la verdad es que dicha destreza se manifiesta solo en aquello que les interesa (jugar, buscar música y películas, relacionarse en las redes sociales...). La mayoría de ellos desconoce muchos de los riesgos del ciberespacio, y no sabe seleccionar con buen criterio las herramientas y metodologías más eficientes para cada tarea escolar o la información más adecuada para cada circunstancia. Por ello, hay que brindar oportunidad para adquirir la formación adecuada y mucho tiempo de práctica. Este es uno de los fundamentos del llamado $\mathrm{Cu}$ rrículum bimodal en el marco de las pedagogías emergentes.

Y ¿qué leer? El auge y la importancia que está adquiriendo la literatura infantil y juvenil invitan a incluir en los currículos y en las programaciones destinadas a los educandos, obras de gran calidad pertenecientes a este corpus, cada vez más numeroso y variado. Se abre un nuevo debate en el que se cuestiona el canon literario: lecturas clásicas versus contemporáneas.

Como explica José Antonio Millán (1992)², aludiendo al ensayo que Italo Calvino dedicó a este aspecto, en la actualidad la lectura de los clásicos provoca dos afiliaciones controvertidas: o son exclusivas de una minoría erudita y, por tanto, no debe incluirse su tratamiento en las aulas escolares; o son una referencia tan necesaria e insustituible que debe ser parte ineludible en la formación de los discentes.

Estamos en la línea de Cerrillo (2013), y que ya apuntábamos con antelación, cuando afirma que quizá la raíz del problema sea la falta de competencia lectora plena, pero es que tal vez esta no se alcance en muchos casos hasta la edad adulta. Por lo que coincidimos con él cuando plantea que si un adolescente puede y quiere, ¿por qué no leer obras clásicas? "[...] Eso sí, sin traumas ni requisitorias amenazantes, como pura obra de arte del lenguaje, como obra maestra de la ironía y el humor, para reírse primero y pensar un poco después. Aunque sea largo, aunque sea un tocho y requiera tiempo y esfuerzo" (p. 24).

Aquí debe surgir la maestría y pericia del docente al proporcionar la ocasión de acercarse al mundo de los clásicos, pero no a los de siempre... o sí, pero también con otros para abrir el abanico de posibilidades porque:

[...] en sus historias y en sus textos está contenida buena parte de la cultura y la tradición del mundo, porque son modelos de escritura literaria, porque son una herencia dejada por nuestros antepasados y porque han contribuido a la formación de un imaginario cultural que haya aportado una peculiar lectura del mundo en sus diferentes épocas. (Cerrillo, 2013, p. 18).

Para lectores incipientes, se requerirá de una preparación previa que puede venir de la mano

2 Lingüista, escritor y periodista. 
de lecturas hechas para ellos: lo primero es darles material que les guste y les aficione a la lectura. Cuando hayamos despertado su atención y hayamos hecho lo posible por mejorar su capacidad lectora (con propuestas como las que presentamos aquí), podrán alcanzar su competencia literaria como receptores. Lo deseable es que alcancen su capacidad de emoción y de sentimiento de placer, según los preceptos de la estética de la recepción, en la línea de lo dispuesto por Suárez (2014).

\section{METODOLOGÍA}

\section{Objeto de estudio y contextualización}

El proceso de esta investigación acción se ha concretado tomando como objeto de estudio un aula de un centro de educación de secundaria de la ciudad de Melilla, en España, compuesta por 35 alumnos y situado en un barrio céntrico. Y se ha llevado a cabo, como experiencia piloto, en el último trimestre del curso escolar 2014-2015.

Antes, es pertinente explicar que Melilla es una ciudad española peculiar pues, geográficamente, se encuentra situada en el norte del continente africano y su frontera con Marruecos hace que comparta la idiosincrasia de otras villas con similares características. En tan solo 12 kilómetros cuadrados y 84.509 habitantes, según el último censo de 2014, conviven desde finales del siglo XIX cinco grupos culturales que mantienen sus costumbres $y$, en algunos casos, rasgos lingüísticos propios. Entre los grupos mayoritarios están: el de procedencia peninsular española (desde 1947) que constituye el $60 \%$ de la población total; y el de ascendencia rifeña o bereber, zona próxima a la ciudad, que supone algo menos del $40 \%$, aunque su número va aumentando. Los grupos minoritarios son: judío, gitano e hindú. De ellos destacamos que el primero es sefardita, procedente también de Marruecos y, como apenas supone un $1 \%$ de la población, trata de preservar su tradición con mucho celo. El colectivo hindú, el menos numeroso de todos, procede de la provincia pakistaní de Sind. En este sentido, lo más interesante para el trabajo que presentamos es la variedad de perspectivas culturales desde las cuales analizar los textos literarios clásicos que, sin duda, enriquecen la dinámica del aula y la definen como contexto intercultural.

En cuanto a sus características culturales, el principal rasgo distintivo del alumnado del centro, y del aula de intervención, es el mismo que caracteriza a Melilla, esto es, la variedad de culturas que conviven en ella. La población musulmana cuenta, a su vez, con dos subgrupos con matices diferentes que se deben tener en cuenta: en el primer subgrupo se incluyen miembros de distintas clases sociales que habitan en diferentes barrios, participan de los mismos rasgos que otras comunidades y son también castellanohablantes; y un segundo grupo con graves problemas de integración por su dificultad con el lenguaje, dado que muchos de ellos son analfabetos y emplean, casi exclusivamente en el entorno más familiar y en las relaciones con los vecinos, una lengua materna distinta al español denominada tamazight, lo cual lleva a una situación de diglosia. Este panorama va aparejado a otros aspectos sociales: vivir en barrios marginales y ocupar puestos de trabajo precario o de economía sumergida. Hay que destacar también como característica la escasa motivación para el estudio y el bajo rendimiento académico que este subgrupo presenta.

El alumnado del centro, y del aula objeto de estudio, pertenece a la clase media y destaca por tener motivación suficiente para proseguir sus estudios, aunque también se incluye en ella un porcentaje de alumnos, del subgrupo al que aludíamos, con dificultades en su escolarización y convivencia $^{3}$ para los que trabajar con una metodología más activa puede resultar mucho más motivador.

En cuanto a infraestructuras y organización del centro, este sigue la normativa vigente y cuenta con instalaciones y recursos suficientes y adecuados.

3 Información extraída del Proyecto de Centro, 2009. 


\section{Metodología didáctica}

Brevemente se puede definir la metodología didáctica como el conjunto de estrategias con base científica que el docente propone en su aula para que los estudiantes adquieran determinados aprendizajes (Fortea, 2009). En el presente programa de intervención, se emplea una metodología activa participativa que convierte a los alumnos en participantes activos en la construcción del conocimiento y rechaza su tradicional posición de simples receptores pasivos; asimismo se otorga relevancia al aprendizaje cooperativo que promueve el uso didáctico de equipos reducidos y heterogéneos de alumnos en actividades de forma que se asegure al máximo la participación igualitaria y se potencie la interacción simultánea entre ellos (Pujolàs, 2009). Se puede afirmar, por tanto, que se propone un modelo de enseñanza/aprendizaje basado en el constructivismo, lo que implica la asunción de una serie de principios pedagógicos cuyo fin es asegurar la construcción de aprendizajes significativos basados en los siguientes principios:

1. Partir de los conocimientos previos de los alumnos: al tratar nuevos conceptos, es imprescindible tener en cuenta los conocimientos previos de los alumnos, ya que la clave para un aprendizaje significativo reside, precisamente, en el vínculo que se establece entre los nuevos conocimientos y las ideas ya presentes en la estructura cognitiva de los individuos.

2. Orientar y reconducir el proceso de los alumnos: además de motivar y propiciar unas condiciones óptimas para el aprendizaje, la labor del docente es orientar y guiar al alumno en su proceso particular de construcción de conocimientos.

3. Integrar activamente al alumno en el proceso de enseñanza/aprendizaje, potenciando el aprendizaje autónomo: en este proceso de enseñanza/ aprendizaje el alumno es quien construye el conocimiento, quien aprende, por tanto, el docente debe guiarle y proporcionarle todos los recursos necesarios para que, de manera autónoma, adquiera las estrategias adecuadas para alcanzar sus objetivos. Además, este papel activo del alumno está vinculado a su actividad mental, por ello, no solo es activo cuando trabaja en alguna actividad, sino también cuando escucha o lee.

4. Favorecer la interacción profesor/alumno y alumno/alumno: la intervención del docente en el aprendizaje es imprescindible para que se produzca con éxito, de manera que una buena comunicación entre profesor y alumno facilita el andamiaje. Por otro lado, la interacción alumno/ alumno favorece el intercambio de información y de diferentes puntos de vista que enriquecen los procesos de aprendizaje; además dicha interacción contribuye a la sociabilización de los educandos. Esta interacción podrá favorecerse desde el mundo digital.

5. Elevar la motivación del alumno con contenidos y actividades próximos a sus intereses y planteando retos alcanzables: el principal requisito para que se produzca el aprendizaje es que los alumnos quieran aprender, por consiguiente se les debe motivar con actividades atractivas y útiles, que no se escapen de sus posibilidades y que se acerquen lo máximo posible a su realidad, una realidad plagada de soportes y otros lenguajes del ciberespacio.

6. Centrar la acción educativa en una atención individualizada: esto permite al docente prevenir dificultades de aprendizaje en los alumnos y poner en práctica mecanismos de refuerzo tan pronto como se detecten dificultades; asimismo, hace posible el desarrollo satisfactorio de aquellos alumnos con mayores capacidades.

La programación mensual del centro para el aula permitió registrar las acciones de línea gruesa y plantear una secuencia de pasos para desarrollar el trabajo de esta investigación que dura dos semanas.

A modo de ejemplo

En sintonía con todo lo expuesto, ofrecemos un ejemplo de propuesta de trabajo realizada en el 
aula que, como ya hemos adelantado, forma parte de una investigación acción más amplia que será puesta en práctica el próximo curso, precisamente, porque los resultados de la experiencia piloto realizada en el último trimestre del curso 20142015 han sido muy esperanzadores.

El texto clásico elegido ha sido Yerma, del escritor Federico García Lorca. Exponemos unas breves pinceladas explicativas para que el lector cuente con mayor información y pueda contextualizar tal propuesta.

\section{La obra}

Yerma (1934) es una obra teatral, escrita en tres actos de dos cuadros cada uno, que desarrolla una tragedia de ambiente rural en los años 1930. Forma parte de la trilogía lorquiana, junto con La casa de Bernarda Alba (1936) y Bodas de sangre (1933).

Yerma es una joven casada con Juan, un hombre que su padre eligió para que fuera su esposo y que ella acepta, no por amor, sino para tener hijos como las demás casadas. Sin embargo, este deseo no es compartido por su marido, a quien le importa más velar por la honra y el trabajo. A lo largo de la obra puede comprobarse cómo este tema es la razón de sus discusiones y de un alejamiento cada vez mayor, pues cada uno de ellos se aferra a distintos motivos para vivir.

En escena también aparecen Víctor y María. Él es amigo de la infancia de Yerma y del que se da a entender que es el hombre con el que ella cree que puede tener hijos porque a su lado se siente diferente, pero su honra firme le impide intentarlo con otro que no sea su marido. María es la amiga y confidente de Yerma que le da esperanza y siempre intenta animarla para aliviarla de lo que supone una tortura para ella: su esterilidad.

La obra comienza cuando lleva dos años casada y aún tiene la esperanza de tener hijos. En el cuadro segundo lleva tres años de matrimonio y en el tercero más de cinco. A medida que el tiempo avanza, su esperanza se convierte en angustia y desesperación, hasta considerar que la falta de pasión de su marido es la auténtica causa de su problema. Todo ello presagia el trágico desenlace.

Según Del Rey Poveda (2000) toda tragedia nace de la resistencia al destino y así se manifiesta en Yerma, nombre que vincula a la protagonista con la tierra baldía, sin descendencia, como ella, frente a la fecundidad que la rodea: mujeres con sus hijos, su amiga María que queda embarazada, sus cuñadas... La obsesión de la protagonista la lleva a escudriñar caminos de superstición, de santería, remedios del pueblo más Ilano, hasta enloquecer.

\section{La propuesta}

Para elaborar el esquema de la propuesta didáctica que exponemos, nos hemos basado en el modelo de Claire Kramsch (1993), aunque nos hemos permitido incluir algunas modificaciones y hemos adaptado en sus apartados estrategias, técnicas y actividades sugeridas por varios autores (Cooper, 1990; Cairney, 1992; Solé, 1992) con matices diferentes, amén de utilizar otras propias y originales.

El esquema consta de cinco fases, además de dos apartados dedicados a la metodología y a la evaluación.

\section{Fase de preparación}

Esta es una fase, única y exclusivamente, del profesor que guíe la actividad. No dudamos de la profesionalidad de los educadores, pero hemos querido incluirla para sentar las bases sobre las que esta práctica docente debe sustentarse y para actualizar los conceptos clave que se deben tener en cuenta en la didáctica de la literatura. Consta de cinco descriptores que esquematizamos a continuación:

- Bases teóricas o conceptos implicados: objetivos generales de la didáctica de la literatura, competencia literaria, comprensión lectora, definición de lectores competentes, distintos enfoques didácticos posibles... Siguiendo los preceptos del enfoque bimodal, el profesor, que tiene claro lo que los alumnos deben aprender, prepara y entrega al alumnado a principio de la propuesta de trabajo, dos listas: el listado del vocabulario/ 
conocimientos que deberán memorizar (por ejemplo: características de la Generación del 27, conceptos clave en Lorca...) y el listado de las actividades prácticas que deberán aprender a hacer y que exponemos más adelante.

- Características del grupo con el que se va a trabajar (en este caso destinado a etapas preuniversitarias).

- Selección del texto: esta selección debe ir en función de los intereses del grupo definido anteriormente, teniendo en cuenta que sea lo bastante motivador como para sentirse atraídos e interesados. En esta ocasión hemos basado la propuesta que presentamos en una obra dramática de Federico García Lorca y, más concretamente, en Yerma, por creer que sus temas y aspectos más relevantes son actuales e interesantes para el grupo destinatario.

- Programación: en este apartado, el educador debe programar los objetivos didácticos que persigue, contenidos para trabajar y actividades propuestas, como debe hacerse en cualquier práctica docente que se precie.

- Metodología y evaluación: para definir los criterios metodológicos que guiarán la tarea, así como una valoración de la actividad (sobre este punto volveremos más adelante).

\section{Fase de prelectura}

Esta fase, como bien indica su denominación, debe ser previa a la lectura del texto seleccionado. Tres son los aspectos que deben incluirse:

- Motivación: puede realizarse de distintos modos. Nosotros proponemos la lectura del primer acto para crear la atmósfera de misterio necesaria con el objeto de inferir o realizar suposiciones sobre el argumento.

- Familiarización con la temática del texto seleccionado: con esta actividad se trata de leer en clase fragmentos de los comentarios que, sobre esta obra y su autor, han realizado algunos críticos literarios, previa selección, por supuesto, del educador (por ejemplo, Ruiz Ramón, 1977;
García Posada, 1979, 1996; Edwards, 1983; Doménech, 1985).

- Información sobre expectativas de comprensión: en este apartado se trata de que los estudiantes, con los datos de los que disponen hasta ahora, pronostiquen qué personajes aparecerán y la relación que mantendrán, qué significado tiene el título de la obra en relación con el argumento, inferir la trama, vaticinar el final. También podrán investigar un poco sobre el autor y el género con el que vamos a trabajar, con consultas bibliográficas y navegando por la red en un afán por discernir qué fuentes son fiables y cuáles no, lo cual forma parte, como ya hemos dicho, del currículo bimodal.

3. Fase de lectura en clase

Tras ratificar que vamos a hacer una lectura estética y no meramente informativa (podemos proponer que traten de diferenciarlas intuitivamente), pasamos a la lectura, propiamente dicha, de la obra. Esta lectura puede hacerse de dos maneras: bien en la propia clase o bien en casa (estableciendo unos plazos que han de ser respetados). Este punto será discernido por el propio educador y habrá de obtenerse el compromiso de los estudiantes. Aquí sugerimos que, en las primeras lecturas que se trabajen con este planteamiento o cuando el texto no sea excesivamente largo (como en esta ocasión), la lectura se haga en clase.

Algunas de las actividades que proponemos pueden realizarse en el transcurso de la lectura o al término de esta; otras, como resulta evidente, deberán hacerse al finalizar:

- Debate para aclarar dudas: estas dudas pueden ser de tipo lexical (recurriremos entonces a presentar estrategias y habilidades para la adquisición de vocabulario, por ejemplo: las claves contextuales, homófonos, sinónimos, etc.), de tipo gramatical o de contenido. Todas serán despejadas por el intercambio de opiniones por parte del alumno con alguna que otra actuación aislada del profesor. 
- Comparación con otras obras del autor: si se conocen, ya que contamos con la ventaja de trabajar con un autor bastante divulgado y cuya información es fácil de obtener consultando bibliografía especializada o fuentes de internet.

- Tertulia o debate: con el fin de comparar el texto, una vez conocido, con lo que imaginaron que leerían en un principio, así como opinar sobre el desarrollo de la obra, soluciones al problema de Yerma, papel de los protagonistas, otros posibles finales, etc.

4. Fase de exploración de la historia

En esta fase nos adentramos en la obra, tratando de explorar los temas y aspectos más relevantes, con las siguientes actividades:

- Organizar un mapa gráfico de la historia: contemplando los siguientes puntos: escenario, personajes, problema, acción, resolución y tema. Pueden consultar fuentes en internet para completarlo. Este mapa puede elaborarse en pizarra digital o con alguna herramienta como Prezzi para presentarlo al resto de compañeros.

- Elaborar un esquema jerárquico de los temas que surgen en la obra por orden de importancia o aparición.

- Personajes: elaborar su retrato psicológico, fichas de descripción de cada personaje con los datos que aporta el texto, trazar un sociograma literario según el modelo de Cairney (1992), coloquio sobre qué personaje ha impactado, sorprendido o gustado más y el motivo, etc. La presentación en la pizarra digital puede ser una buena opción.

- Transtextualidad e intertextualidad: relacionar a Yerma con otras protagonistas femeninas literarias (del corpus de obras que hayan leído o trabajado los estudiantes, pertenecientes al propio autor o a otros). La información puede obtenerse, asimismo, indagando en fuentes de la red.

- "¿Qué opinas de...?": juego que invita a debatir opiniones personales sobre los personajes, comparando los puntos de vista de los estudiantes sobre el drama de Yerma.
- Reorganización y expresión oral de los hechos y acontecimientos: exponiendo la opinión sobre la información que aportan los apartados teatrales, las canciones intercaladas en la obra y su función en todo el conjunto, características textuales del teatro aplicadas a este drama, etc.

- Resumen del argumento: proponerles que imaginen que deben contar a algún amigo o compañero el contenido de esta obra, como si se tratara de una película.

\section{Fase de creatividad y ficcionalidad} Aquí damos rienda suelta a la imaginación. Es la fase de la hipótesis y suposición por excelencia, aunque contamos con datos reales ya trabajados en los apartados anteriores. Es interesante para los alumnos ya que desmitifica y desacraliza la obra volviéndola más accesible, puesto que está supeditada a modificaciones e incluso deformaciones, lícitas en esos momentos en el aula. Es el apartado más personal, pues da lugar a la crítica, interpretación y valoración de cada alumno.

- Creamos el lugar: se trata de describir el lugar donde se desarrolla la obra para centrarnos en las posibles influencias que este pueda tener sobre la historia (ambiente rural, aislamiento) y sobre el comportamiento de los personajes.

- El pasado de Yerma: se trata de imaginar e inferir cuál ha sido el pasado a medio y corto plazo de la protagonista para encontrar una explicación (educacional o vital) al desarrollo de la obra.

- El blog de Yerma: con esta actividad perseguimos que el alumno realice un traspaso a otro género literario y soporte distintos al original en el que se escribió la obra. En esta ocasión proponemos la narración. Con ello podremos precisar mejor las diferencias básicas que distinguen a uno de otro. Ambientamos la actividad comentándoles, por ejemplo, que Yerma, para combatir la soledad a la que se ve forzada durante la noche, mientras espera la llegada de Juan, tiene el hábito de escribir en un blog. 
- "¿Qué pensaría...?": en esta actividad planteamos a los alumnos diferentes hipótesis sobre posibles personajes representativos de otras culturas o religiones o en otras situaciones, que establecen contacto con Yerma, para averiguar e imaginar qué posturas tomarían ante el problema de esta mujer. Así proponemos una conversación narrada en estilo directo o indirecto de Yerma con una mujer actual en su misma situación, con una mujer con muchos hijos, una mujer de otra cultura o religión... Esa conversación podría trasladarse a un grupo de WhatsApp integrado por todas estas mujeres y en el que intercambian tales opiniones.

- "¿Qué pasaría si...?": con esta actividad pretendemos que los alumnos elaboren hipótesis sobre los acontecimientos que provocarían posibles finales, como la adopción, por parte de Yerma, del hijo de María o que, en últimas, se produjese el embarazo de Yerma.

- Representamos: elección de una escena significativa para representar, previo consenso de toda la clase.

- Invención de una escena posterior al final de la obra: los alumnos deberán inventar una escena posterior a la del final, bien en forma dialogada con estilo teatral, bien en forma narrativa, que suponga un cierre más específico sobre la suerte de la protagonista.

En toda esta forma de trabajar el papel del profesor experimenta un giro de 180 grados. Según Cairney (1992), se encarga de proporcionar información, escuchar al alumno (fomentando su intervención en detrimento de la del docente), muestra estrategias eficaces de otros lectores competentes, pone en común intuiciones y éxitos, apoya cuando los resultados no son positivos, evalúa críticamente los esfuerzos, introduce nuevos puntos de vista, etc. Y todo ello en un ambiente de confianza y libertad que estimule la creatividad y desarrolle la personalidad.

\section{RESULTADOS}

Si la metodología seguida que acabamos de sugerir se aparta de la forma tradicional de acometer el análisis de un texto, es obvio que la evaluación ha de ser también distinta. Se impone una participación mucho más activa por parte del alumnado que ha de implicarse mucho más en la tarea planteada. La interacción y la puesta en común del grupo son ahora muy importantes. La exposición de las propias respuestas y las de los otros ayudan a construir el significado de la obra. Son así más susceptibles de ser escuchadas o debatidas con argumentaciones críticas e interpretativas, por lo que la metodología ha de ser abierta y flexible.

Según Solé (1992), en este tipo de actividades, la evaluación se basa en la observación, por lo que requiere que el profesor sea muy receptivo. El proceso de la evaluación debe ser sumativo y formativo, no puntual.

Para Colomer y Camps (1991) es necesario obtener información sobre la actitud emocional del lector frente al texto, sobre el grado de adecuación de la lectura con los objetivos planteados, sobre el grado de manejo de las fuentes de consulta escritas, a las que añadimos las halladas en internet, sobre el proceso de construcción del significado de la obra y sobre el grado de control del lector respecto a la comprensión.

Vallés-Arándiga (1991) propone dos aspectos globales para evaluar: una evaluación del producto comprensivo (con pruebas psicopedagógicas que evalúan la comprensión lectora o como evaluación informal de la comprensión lectora no piscométrica) y una evaluación del proceso comprensivo (en las que se evalúen las habilidades involucradas en cada uno de los grados de la comprensión lectora).

Para esta prueba experimental, se han utilizado dos instrumentos de evaluación: el diario personal del investigador y un cuestionario para 
recoger una valoración del proyecto en sí y de la reacción de los estudiantes.

Para el primero tomamos las categorías inductivas definidas por Suárez (2014), cuya síntesis reproducimos:

- Resultados obtenidos.

- Experiencia personal de lectura.

- Interacción con los estudiantes.

- Aspectos emocionales.

Las valoraciones vinculadas a estas categorías han sido muy positivas durante el tiempo empleado en la actividad y en el diario personal del investigador pueden verse testimonios de los estudiantes de los que recogemos una selección representativa e interesante desde la perspectiva de un análisis cualitativo:

Ahora me encanta ir a clase de lengua y literatura porque siento que estoy haciendo cosas y no solo escuchando al profesor. (Estudiante 4).

He descubierto que la literatura puede ser divertida... (Estudiante 9).

Escuchar la vida de los autores, las obras que escribieron, las características... me resultaba muy aburrido y monótono, porque me lo aprendía de memoria pero ahora soy yo la que descubro cosas y consulto con mis compañeros y con el profesor si son interesantes y así lo aprendo mejor, y seguro que no se me olvidará. (Estudiante 12).

Siempre había pensado que los cuentos eran solo para niños, pero ahora veo que me equivocaba y que me ha gustado leerlos. (Estudiante 30).

Me gusta mucho compartir con mis compañeros la tarea que hemos de hacer porque lo que no se le ocurre a uno, se le ocurre a otro. A veces la interpretación que yo le daba a algo la cambio, porque he escuchado la de otro y veo que no era del todo correcta ni la única que podía hacerse. (Estudiante 23).

Con todas ellas, y muchas más que no se muestran, se detecta el entusiasmo y el despertar de las emociones que han vivido los estudiantes. Del mismo modo, resulta ser una experiencia personal de lectura distinta que puede conducir al descubrimiento y creación del hábito lector. Por otra parte, la clase se convierte así en un lugar de encuentro, de intercambio, de interacción que inyecta un dinamismo que había sido relegado por otras metodologías más tradicionales y ello supone dotarlas de una motivación de la que no puede prescindirse. No hay nada más ilusionante para un docente que sus alumnos declaren la bondad de sus clases y la atracción por aprender y por actuar en ellas. Y esto se torna en un valor añadido cuando se trata de adolescentes cuyo índice de abandono o fracaso suele ser tan alarmante.

Para los otros dos elementos implicados, proyecto y estudiantes, se elaboró un cuestionario, con escala Likert, cuyos criterios se detallan en la tabla 1.

Los resultados obtenidos tras la cumplimentación de este cuestionario por parte de los alumnos se ilustran en el gráfico 1, donde podemos comprobar que la mayoría de las respuestas oscilan entre el "muy satisfactorio" y "satisfactorio".

Los cinco primeros ítems, vinculados al proyecto, registran la pertinencia de este a la vista de los resultados mostrados.

Respecto a los ítems dirigidos al proceso metodológico, confirmamos que se cumplen los principios que guían la intervención y que se han expuesto en el apartado "Metodología didáctica" de este escrito. Así, en el ítem 5 observamos que una amplia mayoría considera que se han activado sus conocimientos previos y que han podido vincularlos con los nuevos, de forma "muy satisfactoria" o "satisfactoria".

Desde el punto de vista social, los resultados de los ítems 6 ("Ha promovido y participado el trabajo grupal con buenos resultados"), 9 ("Asume el punto de vista de los compañeros e incluso modifica el propio con ellos") y 10 ("Ha establecido relaciones culturales y sociales satisfactorias") vislumbran la activación y el desarrollo de esta dimensión con el planteamiento realizado y favorecen la interacción profesor/alumno y alumno/alumno. 
Tabla 1. Resultados de la valoración del proyecto y de la influencia en el estudiante

\begin{tabular}{|c|c|}
\hline CRITERIOS & 1 \\
\hline
\end{tabular}

La progresión de las sesiones ha sido la adecuada.

Ha conseguido el interés y la motivación de los alumnos.

Las actividades planteadas han sido adecuadas.

Ha cumplido con los objetivos propuestos.

\section{ESTUDIANTES}

Ha activado los conocimientos previos y las experiencias y los relacionan con los nuevos conocimientos.

Ha promovido y participado en el trabajo grupal con buenos resultados.

Ha desarrollado capacidades cognitivas y metacognitivas para aprender a aprender y a pensar.

Ha aplicado estrategias orientadas al desarrollo de capacidades comunicativas.

Asume el punto de vista de los compañeros e incluso modifica el propio con ellos.

Ha establecido relaciones culturales y sociales satisfactorias.

$\mathrm{Ha}$ indagado e investigado para ampliar conocimientos.

Ha seleccionado fuentes en internet fidedignas.

Ha mostrado habilidad en el uso de presentaciones digitales.

Muestra implicación y placer en las sesiones.

Fuente: elaboración propia

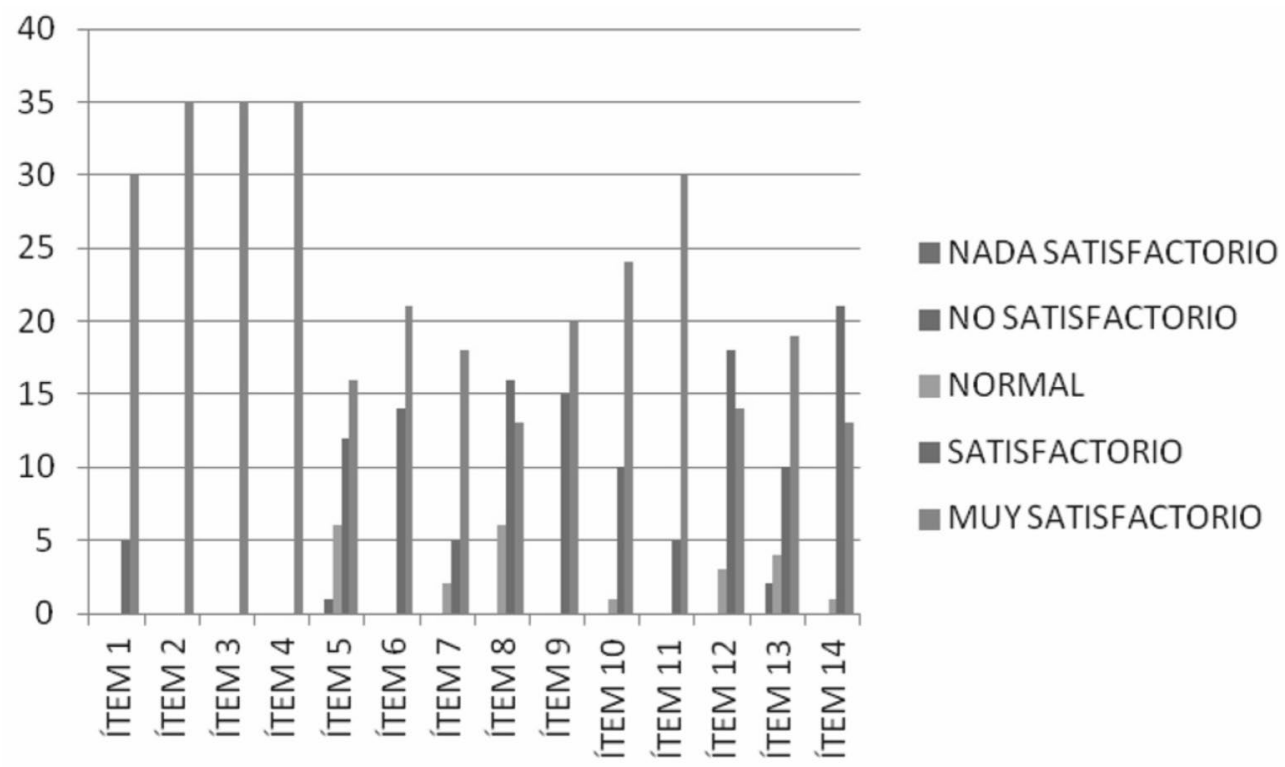

Figura 1. Comparación de porcentajes obtenidos por ítem

Fuente: elaboración propia 
Del mismo modo, también se consigue integrar activamente al alumno en el proceso de enseñanza/aprendizaje y potenciar el aprendizaje autónomo a la vista, sobre todo, de los datos recogidos en los ítems 7 ("Ha desarrollado capacidades cognitivas y metacognitivas para aprender a aprender y a pensar"), 11 ("Ha indagado e investigado para ampliar conocimientos"), 12 ("Ha seleccionado fuentes en internet fidedignas") y 13 ("Ha mostrado habilidad en el uso de presentaciones digitales").

Los datos constatan, además, que se ha elevado la motivación del alumno con contenidos y actividades próximos a sus intereses, y plantean retos alcanzables, según reza en los resultados de los ítems 11 ("Ha indagado e investigado para ampliar conocimientos") y 14 ("Muestra implicación y placer en las sesiones").

Por último, ellos han percibido que su proceso de aprendizaje se ha podido orientar y reconducir, dada la atención individualizada recibida.

En cualquier caso, lo que impera es una evaluación del interés y grado de participación del alumnado, así como el haber llegado a unas conclusiones consensuadas y argumentadas sobre el análisis del texto, tarea que ha resultado exitosa dado el alto grado de aceptación de esta forma de trabajo entre los estudiantes y la calificación positiva obtenida. Este avance de resultados nos permite continuar en esta senda y confirmar la hipótesis de que se trata de una metodología motivadora y eficaz.

\section{CONCLUSIONES}

La primera conclusión a la que se llega tras este primer acercamiento es que merece la pena intentar leer a los clásicos y no hay que desestimarlos, por creer que la distancia lingüística, conceptual o cultural será insalvable. No se trata de hacer eruditos pero sí de ampliar los horizontes y el bagaje de nuestros estudiantes.

Precisamente, cuando el tratamiento metodológico varía, como el expuesto en este trabajo, y el alumno comprueba que le es posible acceder a esta esfera literaria, siente placer y se implica porque su opinión es válida, incluso aunque sea susceptible de mejora o la modifique por la interacción con los demás.

Por otra parte, cuando se facilita la comunicación y transmisión de opiniones, sensaciones, etc., los estudiantes muestran capacidad para relacionar sus conocimientos y experiencias previas para exponer opiniones y construir significados. Son capaces de reflexionar juntos e, incluso, de empatizar con otras opiniones.

Ello conlleva a ampliar conocimientos e interesarse por otros aspectos de otras culturas de su propio entorno, o de entornos más lejanos, con actitud abierta, flexible, respetuosa y tolerante, con todo lo relevante que puede ser en la sociedad donde vivimos.

El interés suscitado entre los estudiantes sin duda los lleva a desarrollar una competencia literaria y más adelante, y tal como matiza Cerrillo (2013):

[...] las obras clásicas no necesitan abogados defensores, solo requieren un hueco en los estantes, en los escaparates y en los planes de estudio. Luego ellas se defienden por sí mismas y se ganan los lectores a pulso, a base de su prestigio histórico, a base de lecturas y relecturas atentas y sensibles a lo largo de los siglos, y son capaces de sembrar de gratitud la mente de sucesivas generaciones por su sola capacidad de emocionar y hacer pensar (p. 18).

\section{RECONOCIMIENTOS}

Este trabajo, que se encuentra en proceso, se enmarca en el Grupo de Investigación en Didáctica de la Lengua y la Literatura (HUM 457) al que pertenece la autora, cuya sede es la Facultad de Ciencias de la Educación de la Universidad de Granada. En concreto, sigue una sus líneas principales de investigación, la literatura y su didáctica, y se centra en el campo de estudio del conocimiento e innovación de los procesos de enseñanza/aprendizaje y de las estrategias didácticas empleadas en nuevas metodologías implementadas en el aula. 


\section{REFERENCIAS}

Cairney, T.H. (1992). Enseñanza de la comprensión lectora. Madrid: Morata.

Camps, A. (1998). La especialidad del área de Didáctica de la Lengua. Una visión sobre la delimitación de los contenidos de la enseñanza de la lengua y la literatura. En: A. Mendoza Fillola (coord.). Conceptos clave en didáctica de la lengua y la literatura (pp. 33-47). Barcelona: Horsori.

Cassany, D. (2012). En_línea: leer y escribir en la red. Colección Argumentos, N. ${ }^{\circ} 41$. Barcelona: Anagrama.

Cerrillo, P. (2013). Canon literario, canon escolar y canon oculto. Quaderns de Filología. Estudis literaris XVIII, 17-31.

Colomer, T. (1999). Introducción a la literatura infantil y juvenil. Madrid: Síntesis.

Colomer, T. y Camps, A. (1991). Ensenyar a llegar, ensenyar a comprendre. Barcelona: Rosa Sensat/Ediciones 62.

Cooper, J.D. (1990). Cómo mejorar la comprensión lectora. Madrid: Visor.

Del Rey Poveda, J.J. (2000). Yerma o el discurso de la intolerancia. Espéculo. Revista de estudios literarios, 14. Universidad Complutense de Madrid. Recuperado de: http://www.ucm.es/info/especulo/numero14/ yerma.html

Doménech, R. (ed.) (1985). La casa de Bernarda Alba y el teatro de García Lorca. Madrid: Cátedra.

Edwards, G. (1983). El teatro de Federico García Lorca. Madrid: Gredos.

Fortea, M.A. (2009). Metodologías didácticas para la enseñanza/aprendizaje de competencias. Trabajo presentado en Curso CEFIRE: "Competencias en el ámbito de las ciencias experimentales. Programar y trabajar por competencias". Castellón. Recuperado de http://cefire.edu.gva.es/pluginfile.php/73850/ mod_folder/content/0/Miguel_A._Fortea/Metodologias_didacticas_E-A_competencias_FORTEA_. pdf?forcedownload $=1$
García Posada, M. (1979). García Lorca. Madrid: Grupo Editorial EDAF.

García Posada, M. (1996). Obras Completas de FGL II. Barcelona: Círculo de Lectores.

Gómez-Villalba B., E. (1995). El reto de hacer lectores. Amigos del Libro. Revista de la Asociación Española del Libro Infantil y Juvenil, 27, 41-52.

Jolibert, J. y Gloton, R. (1985). Context and Culture in Language Teaching. Oxford: Oxford University Press.

Kramsch, C. (1993). Context and Culture in Language Teaching. Oxford: Oxford University Press.

Mendoza, A. (1998). El proceso de recepción lectora. En: A. Mendoza Fillola (coord.). Conceptos clave en didáctica de la lengua y la literatura (pp. 169-189). Barcelona: Horsori.

Millán, J.A. (1992). Porque sí. Calvino y la necesidad de los clásicos. Recuperado de: http://jamillan.com/clasicos. htm\#arriba

Molina-García, M.J. (2002). Bases literarias de la comprensión lectora. Publicaciones, 32, 85-103.

Monson, D. (1989). Rastreando en un libro y más allá de él: las reacciones ante la literatura. En: D. Monson y M. McLenathan (ed.). Crear lectores activos (pp. 7783). Madrid: Visor.

Prensky, M. (2010). Nativos e inmigrantes digitales. Madrid: Institución Educativa SEK. Recuperado el 15 de febrero de 2012 de: http://www.marcprensky.com/ writing/Prensky-NATIVOS\%20E\%20INMIGRANTES\%20DIGITALES\%20\%28SEK\%29.pdf

Puente, A. (1991). Comprensión de la lectura y acción docente. Madrid: Fundación Germán Sánchez Ruipérez.

Pujolàs, P. (2009). La calidad en los equipos de aprendizaje cooperativo. Algunas consideraciones para el cálculo del grado de cooperatividad. Revista de Educación, 349, 225-239.

Ruiz Ramón, F. (1977). Historia del Teatro Español. Siglo XX. Madrid: Cátedra.

Solé, I. (1992). Estrategias de lectura. Barcelona: Graó.

Suárez, V. (2014). La lectura como experiencia estético-literaria. Enunciación 19(2), 215-227.

Vallés, A. (1991). Técnicas de velocidad y comprensión lectora. Madrid: Escuela Española.

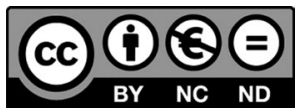

